\title{
Modified Adaptive Resonance Theory Network for Mixed Data Based on Distance Hierarchy
}

\author{
Chung-Chian $\mathrm{Hsu}^{1}$, Yan-Ping Huang ${ }^{1,2}$, and Chieh-Ming Hsiao ${ }^{1}$ \\ ${ }^{1}$ Department of Information Management, National Yunlin University of Science and \\ Technology, 123, Sec. 3, University Road, Douliu, Yunlin 640, Taiwan, R.O.C \\ hsucc@mis.yuntech.edu.tw, g9120817@yuntech.edu.tw \\ ${ }^{2}$ Department of Management Information System, Chin Min Institute of Technology, \\ 110, Hsueh-Fu Road, Tou-Fen, Miao-Li 351, Taiwan, R.O.C \\ sunnyachinmin.edu. tw
}

\begin{abstract}
Clustering of data is a fundamental data analysis step that has been widely studied across in data mining. Adaptive resonance theory network (ART) is an important algorithm in Clustering. ART is also very popular in the unsupervised neural network. Type I adaptive resonance theory network (ART1) deals with the binary numerical data, whereas type II adaptive resonance theory network (ART2) deals with the general numerical data. Several information systems collect the mixing type attitudes, which included numeric attributes and categorical attributes. However, ART1 and ART2 do not deal with mixed data. If the categorical data attributes are transferred to the binary data format, the binary data do not reflect the similar degree. It influences the clustering quality. Therefore, this paper proposes a modified adaptive resonance theory network (M-ART) and the conceptual hierarchy tree to solve similar degrees of mixed data. This paper utilizes artificial simulation materials and collects a piece of actual data about the family income to do experiments. The results show that the M-ART algorithm can process the mixed data and has a great effect on clustering.
\end{abstract}

Keywords: adaptive resonance theory network (ART), distance hier-archy, clustering algorithm, data mining, software engineering.

\section{Introduction}

Clustering is the unsupervised classification of patterns into groups. It is an important data analyzing technique, which organizes a collection of patterns into clusters based on similarity [1-3]. Clustering is useful in several exploratory pattern-analysis, grouping, decision-making, and machine-learning situations. This includes data mining, document retrieval, image segmentation, and pattern classification. Clustering methods have been successfully applied in many fields including image processing [1], pattern recognition [4], biology, psychiatry, psychology, archaeology, geology, geography, marketing and information retrieval [5,6], software engineering [7-10]. Intuitively, patterns with a valid cluster are more similar to each other than they are to a pattern belonging to a different cluster. 
The majority of the software clustering approaches presented in the literature attempt to discover clusters by analyzing the dependencies between software artifacts, such as functions or source files [7-13]. Software engineering principles, such as information hiding or high-cohesion and low-coupling are commonly employed to help determine the boundaries between clusters.

Most of clustering algorithms consider either categorical data or numeric data. However, many mixed datasets including categorical and numeric values existed nowadays. A common practice to clustering mixed dataset is to transform categorical values into numeric values and then proceed to use a numeric clustering algorithm. Another approach is to compare the categorical values directly, in which two distinct values result in distance 1 while identical values result in distance 0 . Nevertheless, these two methods do not take into account the similarity information embedded between categorical values. Consequently, the clustering results do not faithfully reveal the similarity structure of the dataset. This article is based on distance hierarchy [2-3] to propose a new incremental clustering algorithm for mixed datasets, in which the similarity information embedded between categorical attribute is considered during clustering. In the setting, each attribute of the data is associated with a distance hierarchy, which is an extension of the concept hierarchy [21] with link weights representing the distance between concepts. The distance between two mixed data patterns is then calculated according to distance hierarchies.

The rest of this article is organized as follows. Section 2 reviews clustering algorithms and discusses the shortcomings of the conventional approaches to clustering mixed data. Section 3 presents distance hierarchy for categorical data and proposes the incremental clustering algorithm based on distance hierarchies. In Section 4, experimental results on synthetic and real datasets are presented. Conclusions are given in Section 5.

\section{Related Work}

Adaptive resonance theory neural networks model real-time prediction, search, learning, and recognition. ART networks function as models of human cognitive information processing [15-19]. A central feature of all ART systems is a pattern matching process that compares an external input with the internal memory of an active code. ART1 deals with the binary numerical data and ART2 deals with the general numerical data [18]. However, these two methods do not deal with mixed data attributes.

About clustering mixed data attributes, there are two approaches for mixed data. One is resorted to a pre-process, which transferred the data to the same type, either all numeric or all categorical. For transferring continuous data to categorical data, some metric function is employed. The function is based on simple matching in which two distinct values result in distance 1 , with identical values of distance 0 [20]. The other is to use a metric function, which can handle mixed data [21]. Overlap metric is for nominal attributes and normalized Euclidean distance is for continuous attributes.

Among problems with simple matching and binary encoding, a common approach for handling categorical data is simple matching, in which comparing two identical categorical values result in distance 0 , while two distinct values result in distance 1 
$[21,22]$. In this case, the distance between patterns of Gary and John in the previous example becomes $\mathrm{d}($ Gary, John $)=1$, which is the same as $\mathrm{d}(\mathrm{John}$, Tom $)=\mathrm{d}$ (Gary, Tom $)=1$. Obviously, the simple matching approach disregards the similarity information embedded in categorical values.

Another typical approach to handle categorical attributes is to employ binary encoding that transforms each categorical attribute to a set of binary attributes and a categorical value is then encoded to a set of binary values. As a result, the new relation contains all numeric data, and the clustering is therefore conducted on the new dataset. For example, as the domain of the categorical attribute: Favorite_Drink. The set of it is \{Coke, Pepsi, Mocca\}. Favorite_Drink is transformed to three binary attributes: Coke, Pepsi and Mocca in the new relation. The value Coke of Favorite_Drink in a pattern is transformed to a set of three binary values in the new relation, i.e. $\{$ Coke $=1$, Pepsi $=0$, Mocca $=0\}$. The Manhattan distance of patterns Gary and John is $\mathrm{d}_{\mathrm{M}}($ Gary, John $)=2$, which is the same as $\mathrm{d}_{\mathrm{M}}($ Gary, Tom $)$ and $\mathrm{d}_{\mathrm{M}}(\mathrm{John}$, Tom), according to the new relation. Traditional clustering algorithm transfers Favorite_Drink categorical attributes into a binary numerical attribute type as shown in figure 1.

\begin{tabular}{|l|c|c|}
\hline ID & Favorite Drink & Amt. \\
\hline Gary & Coke & 70 \\
\hline John & Pepsi & 70 \\
\hline Tom & Coffee & 70 \\
\hline
\end{tabular}

\begin{tabular}{|l|l|l|l|l|}
\hline ID & Coke & Pepsi & Coffee & Amt. \\
\hline Gary & 1 & 0 & 0 & 70 \\
\hline John & 0 & 1 & 0 & 70 \\
\hline Tom & 0 & 0 & 1 & 70 \\
\hline
\end{tabular}

Fig. 1. Traditional clustering algorithm transfers Favorite_Drink categorical attributes into binary numerical attribute type

The ART network is a popular incremental clustering algorithm [1]. It has several variants $[23,24]$, in which ART1 handles only the binary data and ART2 can handle only the arbitrary continuous data. K-prototype [25] is a recent clustering algorithm for mixed data. It transfers categorical data attributes to the binary data format, however, the binary data do not reflect the similar degree. It influences the clustering quality. Therefore, this paper proposes a modified adaptive resonance theory network algorithm and the conceptual hierarchy tree to solve the similar degree of mixed data.

\section{Clustering Hybrid Data Based on Distance Hierarchy}

The distance hierarchy tree is a concept hierarchy structure. It is also a better mechanism to facilitate the representation and computation of the distance between categorical values. A concept hierarchy consists of two parts: a node set and a link set $[2,3,26,27]$. According to binary encoding approach, it does not reflect the similar degree. However, it influences the clustering quality. Maintenance was difficult when the domain of a categorical attribute changes, because the transformed relation schema also needs to be changed. The transformed binary attributes cannot preserve the semantics of the original attribute. Because of the drawbacks resulting from the binary-encoding approach, this paper uses distance hierarchy to solve the similar degree of mixed data. 
This paper extends the distance hierarchy structure with link weights. Each link has a weight representing a distance. Link weights are assigned by domain experts. There are several assignment alternatives. The simplest way is to assign all links as a uniform constant weight. Another alternative is to assign heavier weights to the links closer to the root and lighter weights to the links away from the root. For simplicity, unless stated explicitly, each link weight is set to 1 in this article. The distance of two concepts at the leaf nodes is the total weight between those two nodes.

A point $\mathrm{X}$ in a distance hierarchy consists of two parts, an anchor and a positive real-value offset, denoted as $\mathrm{X}(\mathrm{N}, \mathrm{d})$, that is, $\operatorname{anchor}(\mathrm{X})=\mathrm{N}$ and $\operatorname{offset}(\mathrm{X})=\mathrm{d}$. The anchor is a leaf node and the offset represents the distance from the root of the hierarchy to the point. A point $\mathrm{X}$ is an ancestor of $\mathrm{Y}$ if $\mathrm{X}$ is in the path from $\mathrm{Y}$ to the root of the hierarchy. If neither one of the two points is an ancestor of the other point, then the least common ancestor, denoted as $\operatorname{LCA}(\mathrm{X}, \mathrm{Y})$, is the deepest node that is an ancestor of $\mathrm{X}$ as well as $\mathrm{Y}$.

A special distance hierarchy calls numeric distance hierarchy for a numeric attribute, say $\mathbf{x}_{\mathrm{i}}$, is a degenerate one, which consists of only two nodes, a root MIN and a leaf MAX, and has the link weight $w$ being the domain range of $\mathbf{x}_{\mathrm{i}}$, i.e. $\mathrm{w}=$ $\left(\max _{\mathrm{i}}-\min _{\mathrm{i}}\right)$. A point $\mathrm{p}$ in such a distance hierarchy has the value $\left(\mathrm{MAX}, \mathrm{d}_{\mathrm{p}}\right)$ where the anchor is always the MAX and the offset $d_{p}$ is the distance from the point to the root MIN.

About measuring distance, the distance between two data points can be measured as follows: Let $\mathrm{x}=\left[\begin{array}{llll}\mathrm{x}_{1} & \mathrm{x}_{2} & \ldots & \mathrm{x}_{\mathrm{n}}\end{array}\right]$ and $\mathrm{y}=\left[\begin{array}{llll}\mathrm{y}_{1} & \mathrm{y}_{2} & \ldots & \mathrm{y}_{\mathrm{n}}\end{array}\right]$. The distance between a training pattern $\mathbf{x}$ and an M-ART neuron $\mathbf{y}$ is measured as the square root of the sum of the square differences between each-paired components of $\mathbf{x}$ and $\mathbf{y}$. Specifically, $\mathbf{x}$ and $\mathbf{y}$ represent a training data and a map neuron, respectively, with n-dimension, and $\mathrm{C}$ is a set of $\mathrm{n}$ distance hierarchies, then the distance between $\mathbf{x}$ and $\mathbf{y}$ can be expressed as

$$
d(\mathbf{x}, \mathbf{y})=\|\mathbf{x}-\mathbf{y}\|=\left(\sum_{i=1, n} w_{i}\left(x_{i}-y_{i}\right)^{2}\right)^{1 / 2}=\left(\sum_{i=1, n} w_{i}\left(h\left(x_{i}\right)-h\left(y_{i}\right)\right)^{2}\right)^{1 / 2}
$$

Where $h\left(x_{i}\right)$ and $h\left(y_{i}\right)$ are the mapping of $x_{i}$ and $y_{i}$ to their associated distance hierarchy $h_{i}$ and $w_{i}$, the attribute weight, is a user specified parameter allowing the domain expert to give different weights. For a numeric attribute $I, h\left(x_{i}\right)-h\left(y_{i}\right)$ is equal to $\mathrm{x}_{\mathrm{i}}-\mathrm{y}_{\mathrm{i}}$, since $\mathrm{h}\left(\mathrm{x}_{\mathrm{i}}\right)-\mathrm{h}\left(\mathrm{y}_{\mathrm{i}}\right)=\left(\mathrm{MIN}, \mathrm{d}_{\mathrm{h}(\mathrm{xi})}\right)-\left(\mathrm{MIN}, \mathrm{d}_{\mathrm{h}(\mathrm{yi})}\right)=\left(\mathrm{MIN}, \mathrm{x}_{\mathrm{i}}-\min _{\mathrm{i}}\right)-\left(\mathrm{MIN}, \mathrm{y}_{\mathrm{i}}-\right.$ $\left.\min _{\mathrm{i}}\right)=\left(\mathrm{x}_{\mathrm{i}}-\mathrm{y}_{\mathrm{i}}\right)$.

This paper proposes the distance hierarchy tree structure to overcome the expression for similar degree. This distance hierarchy tree algorithm combines the adaptive resonance theory network algorithm and it can be effective with mixed data in data clustering. This section presents distance hierarchy for categorical data and it proposes the incremental clustering algorithm based on distance hierarchies.

The categorical utility function [28] attempts to maximize the probability that the two objects in the same cluster have attribute values in common and the probability that the objects from different clusters have different attributes. The categorical utility of a set of clusters can be calculated as

$$
\mathrm{CU}=\sum_{\mathrm{k}}\left(\frac{\left|\mathrm{C}_{\mathrm{k}}\right|}{|\mathrm{D}|} \sum_{\mathrm{i}} \sum_{\mathrm{j}}\left[\mathrm{P}\left(\mathrm{A}_{\mathrm{i}}=\mathrm{V}_{\mathrm{ij}} \mid \mathrm{C}_{\mathrm{k}}\right)^{2}-\mathrm{P}\left(\mathrm{A}_{\mathrm{i}}=\mathrm{V}_{\mathrm{ij}}\right)^{2}\right]\right.
$$

Here, $P\left(A_{i}=V_{i j} \mid C_{k}\right)$ is the conditional probability that the attribute $i$ has the values $V_{i j}$ given the cluster $C_{k}$, and $P\left(A_{i}=V_{i j}\right)$ is the overall probability of the attribute $i$ having 
the values $V_{i j}$ in the entire set. The function aims to measure if the clustering improves the likelihood of similar values falling in the same cluster. Obviously, the higher the CU values, the better the clustering result [29].

For numeric attributes, the standard deviation represents the dispersion of values. Variance $\left(\sigma^{2}\right)$ can be used for evaluating the quality of clustering numeric data. Several cluster validity indices, such as Davies-Bouldin (DB) Index and Calinski Harabasz $(\mathrm{CH})$ Index [27, 30], have been published; however, they are only suitable for the numeric data. Hence, in order to evaluate the effectiveness of clustering mixed data, this paper uses CV index [31], which combined the category utility (CU) function with variance. The $\mathrm{CV}$ is defined as in Equation (3), where the $\mathrm{CU}$ and variance are the validity index for categorical and numeric data, respectively. The higher the $\mathrm{CV}$ values, the better the clustering result.

$$
C V=\frac{C U}{1+\text { Variance }\left(\sigma^{2}\right)}
$$

\section{Experiments}

This paper develops a prototype system with Borland $\mathrm{C}++$ Builder 6. A series of experiments have been performed in order to verify the method. A mixed synthetic dataset and a UCI dataset have also been designed to show the capability of the MART in reasonably expressing and faithfully preserving the distance between the categorical data. It also reports the experimental results of artificial and actual data.

These experiments use a real Adult dataset from the UCI repository with 48,842 records of 15 attributes, including eight categorical attributes, six numerical attributes, and one class attribute.

This experiment uses 7 attributes, which include three categorical attributes, such as Relationship, Marital_status, and Education; and four numeric attributes, Capital_gain, Capital_loss, Age, and Hours_per_week. The concept hierarchies are constructed in figure 2 .

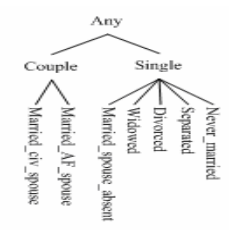

( a) marital-status

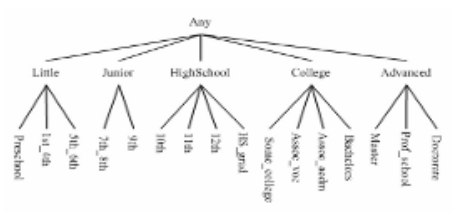

(b) education

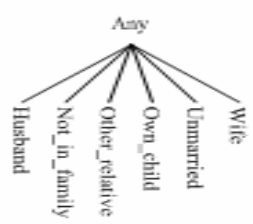

( c) relationship

Fig. 2. Concept hierarchies for (a) Marital-status (b) Education and (c) Relationship attributes of the Adult dataset

The M-ART parameters are established as follows: the initial warning value is 0.55 and it increases progressively 0.05 until 0.75 . The initial learning rate is 0.9 . The stop condition t occurs when the momentum of the output layer is lower than 0.000015 .

This paper collects the dataset with different methods. These methods divide adult datasets into 5, 6, 7 and 8 groups. Concerning the $\mathrm{CU}$ values for categorical attributes, 
the higher the $\mathrm{CU}$ values, the better the clustering result. The $\mathrm{CU}$ value of clustering M-ART method is the highest, K-prototype method is second, and traditional ART2 is the lowest. The symbol "***" means that it does not find the suitable parameter to divide into group with the datasets. The parameter of ART2 reaches 7, it is unable to divide seven groups all the time. The problem occurs because there are too many parameters in ART2.

This paper normalizes the variance between 0 and 1 for numeric results. The normalized variance is useful in CV index. Table 1 shows the $\mathrm{CV}$ values of the clustering results by M-ART, ART2 and k-prototypes on level 1 and the leaf level in individual concept hierarchies with cluster numbers 5, 6, 7 and 8. The higher the value of $\mathrm{CV}$ values, the better the clustering result. The CV value in M-ART method is the highest, K-prototype method is second, and traditional ART2 is the lowest.

Table 1. The CV values for Adult dataset with 5, 6, 7, 8 clusters by M-ART, ART2 and KPrototypes

\begin{tabular}{|c|c|c|c|c|c|c|c|c|c|}
\hline \multicolumn{10}{|c|}{ M-ART } \\
\hline \multirow{2}{*}{$\begin{array}{l}\text { Cluster } \\
\text { No. }\end{array}$} & \multicolumn{2}{|c|}{$\mathrm{CU}$} & \multicolumn{3}{|c|}{ Variance } & & \multicolumn{2}{|l|}{$\mathrm{CV}$} & \multirow{2}{*}{ Increased } \\
\hline & Leaf & Level 1 & age & gain & loss & hrs_per_week & Leaf_Level & $\begin{array}{l}\text { Level } 1 \\
\end{array}$ & \\
\hline 5 & 1.069 & 1.16 & 0.127 & 0.022 & 0.038 & 0.071 & 0.85 & 0.59 & $31.08 \%$ \\
\hline 6 & 1.113 & 1.2 & 0.163 & 0.023 & 0.044 & 0.085 & 0.85 & 0.60 & $29.05 \%$ \\
\hline 7 & 1.115 & 1.21 & 0.182 & 0.011 & 0.044 & 0.100 & 0.83 & 0.61 & $27.05 \%$ \\
\hline 8 & 1.177 & 1.31 & 0.218 & 0.014 & 0.052 & 0.115 & 0.84 & 0.65 & $23.00 \%$ \\
\hline
\end{tabular}

\begin{tabular}{|c|c|c|c|c|c|c|c|c|c|}
\hline \multicolumn{10}{|c|}{ K-Prototype } \\
\hline \multirow{2}{*}{$\begin{array}{l}\text { Cluster } \\
\text { No. }\end{array}$} & \multicolumn{2}{|c|}{$\mathrm{CU}$} & \multicolumn{3}{|c|}{ Variance } & & \multicolumn{2}{|l|}{$\mathrm{CV}$} & \multirow{2}{*}{ Increased } \\
\hline & Leaf & Level 1 & age & gain & loss & hrs_per_week & Leaf_Level & Level 1 & \\
\hline 5 & 0.859 & 0.834 & 0.114 & 0.033 & 0.046 & 0.073 & 0.68 & 0.46 & $32.85 \%$ \\
\hline 6 & 1.002 & 0.977 & 0.424 & 0.72 & 1.454 & 2.937 & 0.15 & 0.16 & $-1.72 \%$ \\
\hline 7 & 1.039 & 0.919 & 0.515 & 0.893 & 1.801 & 3.635 & 0.13 & 0.12 & $7.01 \%$ \\
\hline 8 & 1.088 & 1.087 & 0.610 & 1.068 & 2.154 & 4.342 & 0.12 & 0.13 & $-5.56 \%$ \\
\hline
\end{tabular}

\begin{tabular}{|c|c|c|c|c|c|c|c|c|c|}
\hline \multicolumn{10}{|c|}{$\overline{\text { ART2 }}$} \\
\hline \multirow{2}{*}{$\begin{array}{l}\text { Cluster } \\
\text { No. }\end{array}$} & \multicolumn{2}{|l|}{$\mathrm{CU}$} & \multicolumn{3}{|c|}{ Variance } & & \multicolumn{2}{|l|}{$\mathrm{CV}$} & \multirow{2}{*}{ Increased } \\
\hline & Leaf & Level 1 & age & gain & loss & hrs_per_week & Leaf_Level & Level 1 & \\
\hline 5 & 0.0010 & 0.00081 & 0.176 & 0.027 & 0.042 & 0.079 & 0.00075 & 0.00070 & $6.64 \%$ \\
\hline 6 & 0.0043 & 0.00570 & 0.211 & 0.033 & 0.051 & 0.095 & 0.00309 & 0.00482 & $-55.87 \%$ \\
\hline 8 & 0.0073 & 0.00757 & 0.281 & 0.044 & 0.068 & 0.128 & 0.00479 & 0.00608 & $-26.71 \%$ \\
\hline
\end{tabular}

\section{Conclusions and Future Work}

Most traditional clustering algorithms can only handle either categorical or numeric value. Although some research results have been published for handling mixed data, they still cannot reasonably express the similarities among categorical data.

This paper proposes the distance hierarchy tree structure to overcome the expression for similar degree. This distance hierarchy tree algorithm combines the adaptive resonance theory network algorithm and it can be effective with mixed data in data clustering. It presents a MART algorithm, which can handle mixed dataset 
directly. The experimental results on synthetic data sets show that the proposed approach can better reveal the similarity structure among data, particularly when categorical attributes are involved and have different degrees of similarity, in which the traditional clustering approaches do not perform well. The experimental results on the real dataset have better performances than other algorithms.

MART is a clustering algorithm for any field with mixed data. The future work will try to use this method in finding out the pattern rules from software engineering databases

\section{References}

1. Jain, A., and Dubes, R.: Algorithms for clustering Data. Prentice-Hall, Englewood Cliffs. NJ. (1988).

2. Hsu, C. C.: Generalizing Self-Organizing Map for Categorical Data. IEEE Transactions on Neural Networks. (2006, In press).

3. Hsu, C. C. and Wang, S. H.: An Integrated Framework for Visualized and Exploratory Pattern Discovery in Mixed Data. IEEE Transactions on Knowledge and Data Engineering. vol. 18, no. 2. (2006) 161-173.

4. Anderberg, M.: Cluster Analysis for Applications. Academic Press, New York. (1973).

5. Rasmussen, E.: Clustering Algorithms. Information Retrieval: Data Structures \& Algorithms. William B. Frakes and Ricardo Baeza-Yates (Eds.). Prentice Hall. (1992).

6. Salton, G. and Buckley, C.: Automatic Text Structuring and Retrieval-experiments in Automatic Encyclopedia Searching. Proceedings of the Fourteenth International ACM SIGIR Conference on Research and Development in Information Retrieval. (1991) 21-30.

7. Kadamuddi, D. and Tsai, J. P.: Clustering algorithm for parallelizing software systems in multiprocessors environment. Software Engineering, IEEE Transactions, vol. 26, Issue 4, (2000) 340-361.

8. Tian, J.: Better reliability assessment and prediction through data clustering. Software Engineering, IEEE Transactions, vol. 28, Issue 10, (2002) 997-1007.

9. Chen K., Zhang W., Zhao H., Mei H.: An approach to constructing feature models based on requirements clustering. Requirements Engineering, 2005. Proceedings. 13th IEEE International Conference. (2005) 31-40.

10. Andritsos, P. and Tzerpos, V.: Information-theoretic software clustering. Software Engineering, IEEE Transactions, vol. 31, Issue 2. (2005) 150-165.

11. Hutchens, D. H. and Basili, V.R.: System Structure Analysis: Clustering with Data Bindings. Software Engineering, IEEE Transactions, vol. 11, no. 8. (1985) 749-757.

12. Schwanke, R. W.: An Intelligent Tool for Re-Engineering Software Modularity. Proc. 13th Int'l Conf. Software Engineering. (1991) 83-92.

13. Mancoridis, S., Mitchell, B., Chen, Y. and Gansner, E.: Bunch: A Clustering Tool for the Recovery and Maintenance of Software System Structures. Proc. Int'l Conf. Software Maintenance. (1999).

14. Can, F.: Incremental clustering for dynamic information processing. ACM Transaction for Information Systems, vol. 11. (1993) 143-164.

15. Carpenter, G. A.: Distributed learning, recognition, and prediction by ART and ARTMAP neural networks. Neural Networks, vol. 10, no. 8. (1997) 1473-1494.

16. Carpenter, G. A., and Grossberg, S.: Normal and amnesic learning, recognition, and memory by a neural model of cortico-hippocampal interactions. Trends in Neuroscience, vol. 16, no. 4. (1993) 131-137. 
17. Grossberg, S.: How does a brain build a cognitive code? Psychological Review, vol. 87. (1980) 1-51.

18. Grossberg, S.: The link between brain, learning, attention, and consciousness. Consciousness and Cognition, vol. 8. (1999) 1-44.

19. Grossberg, S.: How does the cerebral cortex work? Development, learning, attention, and 3D vision by laminar circuits of visual cortex. Behavioral and Cognitive Neuroscience Reviews, vol. 2, no. 1. (2003) 47-76.

20. Guha, S., Rastogi, R. and Shim, K.: ROCK: A robust clustering algorithm for categorical attributes. Proceedings of the IEEE Conference on Data Engineering. (1999) 512-521.

21. Wilson, D.R. and Martinez, T. R.: Improved heterogeneous distance functions. Journal of Artificial Intelligence Research, vol. 6. (1997) 1-34.

22. Ester, M., Kriegel, H. P., Sander, J., Wimmer, M. and Xu, X.: Incremental clustering for mining in a data warehousing environment. Proceedings of the 24th Intl. Conf. on Very Large Data Bases (VLDB). (1998) 323-333.

23. Carpenter, G. A. and Grossberg, S.: ART 2 : Self-organization of stable category recognition codes for analog input patterns. Applied Optics : Special Issue on Neural Networks, vol. 26. (1987) 4919-4930.

24. Carpenter, G., Grossberg, A., S. and Rosen, D. B.: Fuzzy ART: fast stable learning and categorization of analog patterns by an adaptive resonance system. Neural Networks, vol. 4. (1991) 759-771.

25. Huang, Z.: Extensions to the k-means algorithm for clustering large data sets with categorical values. Data Mining and Knowledge Discovery, vol. 2, no. 3. (1998) 283-304.

26. Dash, M. and Choi, K., Scheuermann, P. and Liu, H.: Feature selection for clustering - a filter solution, IEEE International Conference on Data Mining. (2002) 115 - 122.

27. Maulik, U. and Bandyopadhyay, S.: Performance evaluation of some clustering algorithms and validity indices. IEEE Transactions on Pattern Analysis and Machine Intelligence. (2002) 1650-1654.

28. Gluck, M. A. and Corter, J. E.: Information, uncertainty, and the utility of categories. Proceedings of the Seventh Annual Conference of the Cognitive Science Society. (1985).

29. Barbara, D., Couto, J. and Li, Y.: COOLCAT: an entropy-based algorithm for categorical clustering. Proceedings of the eleventh international conference on Information and knowledge management. (2002) 582-589.

30. Halkidi, M., Batistakis, Y. and Vazirgiannis, M.: On clustering validation techniques. Journal of Intelligent Information Systems, vol. 17. (2001) 107-145.

31. Hsu, C. C. and Chen, Y. C.: Mining of Mixed Data with Application to Catalog Marketing. Expert Systems with Applications. (2006, In press). 